\title{
Musculoskeletal ultrasound: an effective tool to help medical students improve joint inflammation detection?
}

\author{
Aníbal Ruiz-Curiel ${ }^{1}$, María Dolores Díaz-Barreda1, Marta González-Rodríguez ${ }^{1}$, \\ Teresa Martín-Delgado ${ }^{1}$, Adriana Medrano-Antoñazas', Raquel Muñoz-González ${ }^{1}$, \\ Silvia Perales-Torres ${ }^{1}$, Javier Sánchez-Montero' ${ }^{1}$, Iustina Janta ${ }^{2}$, Juan Carlos Nieto², \\ Luis Collado-Yurrita ${ }^{3 *}$, Francisco Javier López-Longo ${ }^{2 *}$, Esperanza Naredo ${ }^{2 *}$
}

${ }^{1}$ Faculty of Medicine, Universidad Complutense, ${ }^{2}$ Department of Rheumatology, Hospital General Universitario Gregorio Marañón, ${ }^{3}$ Department of Medicine, Faculty of Medicine, Complutense University, Madrid, Spain

*Senior authors

\begin{abstract}
Aim: The objective of this study was to evaluate whether musculoskeletal (MS) ultrasound (US) can be useful in helping medical students to detect joint inflammation through physical examination. Material and methods: The study was performed by two groups of four $6^{\text {th }}$ year medical students. None had received any previous training in the clinical examination of joints or the use of ultrasound. Students were put through a 5-session training programme on the clinical detection of either knee [group 1] or metacarpophalangeal (MCP) [group 2] inflammation. After an initial training session on physical examination of normal and inflamed joints, the students examined 170 joints from 41 patients attending the hospital outpatient clinic in 4 separate sessions. The same joints were assessed for synovitis with US with the ensuing data compared to that of the students and analyzed for concordance with Cohen's unweighted kappa. Results: In total 60 knees [group 1] and 110 MCP [group 2] were evaluated. The agreement between the presence of arthritis detected by the students in the four sessions and the presence of synovitis detected by US improved from the session I to sessions III with a marked improvement in the last session. Conclusions: MSUS may be an effective technique for helping students to acquire the ability to detect joint inflammation.

Keywords: medical students, musculoskeletal ultrasound, education, training, arthritis
\end{abstract}

\section{Introduction}

For the sixth year students of medicine there is no current training on detecting inflamed joints through physical examination. The scope of this paper was to examine whether musculoskeletal ultrasound (MSUS) might be useful as a diagnostic tool to help students acquire competence and skills in this area.

This exercise was conducted in conjunction with experienced rheumatologists, for whom joint inflammation

Received 14.04.2016 Accepted 20.05.2016

Med Ultrason

2016, Vol. 18, No 3, 294-298

Corresponding author: Iustina Janta

Department of Rheumatology

Hospital General Universitario Gregorio Marañón

Doctor Esquerdo, 46, 28007 Madrid, Spain

E-mail: iustinajanta@yahoo.com or arthritis is the most characteristic clinical manifestation of many rheumatic diseases. For these doctors the ability to identify inflammation in symptomatic joints is paramount. This is achieved through external observation and palpation, with pathologic joints determined through abnormalities to anatomical structures such as soft tissue under or between the bones, liquid matter, tenderness, swelling or warmth. However, even for experts, the clinical detection of joint inflammation through physical examination is not always easy, evidenced by relatively low peer consensus or interobserver reliability, estimated at 0.29 and 0.98 [1-3].

The diagnosis process can be improved, however, through MSUS which is a diagnostic imaging technique based on the use of ultrasound to view internal structures. Employed in many countries, including Spain, the technique is more sensitive and accurate than the physical 


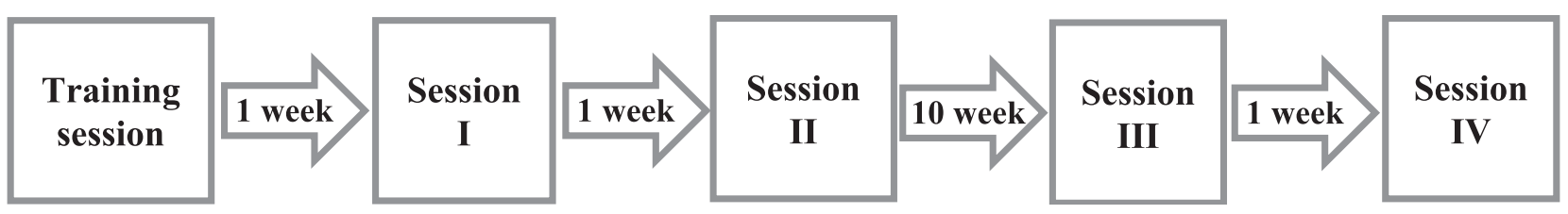

Fig 1. Training process

examination in detecting inflammation in limb joints $[4,5]$. Furthermore, MSUS has proven to be a useful tool in helping medical undergraduates broaden their understanding of anatomy [6-8]. Its use has extended to areas other than rheumatology to enable physicians another perspective on internal structures. Additionally, MSUS can also be used by the patient to self-assess joint inflammation [9].

To the best our knowledge, there are no training methods or studies reported which evaluate MSUS as a teaching tool to aid medical students in the clinical detection of joint inflammation [3].

We hypothesized that the complementary use of MSUS as a diagnostic tool could help medical students to distinguish normal joints from those presenting arthritis. Therefore, the aim of this study was to test whether MSUS is effective as a teaching tool.

\section{Material and methods}

\section{Study design}

This study was conducted by the Department of Rheumatology, University Hospital Gregorio Marañón, Madrid, Spain, from September to December 2015. It was approved by the Ethics Committee of Clinical Investigation and conducted in accordance with the Declaration of Helsinki. All patients who participated signed the informed consent.

Two groups of $6^{\text {th }}$ year medical students of the Complutense University, Madrid (Spain) conducted the study. Each group comprised 4 students -1 male and 3 female in both cases - none of whom had any previous training in joint or ultrasound examination.

The study consisted of five sessions as follows:

a) Training session: a 2-hour training session was given on the clinical detection of inflammation in either the knee [group 1] or metacarpophalangeal (MCP) [group 2] joints.

Firstly, the students performed knee [group 1] and MCP [group 2] physical examinations on each other to enable them to appraise healthy joints.

Students were then trained by consultant rheumatologists (IJ, JCN) in how to detect inflammation in target joints through physical examination. This was demon- strated with rheumatic patients attending the outpatient clinic on that particular day presenting varying degrees of clinical inflammation.

b) Sessions I to $I V$ : the first session was conducted one week after the training session with the second conducted one week following the first. A 10-week break ensued to prevent any possible influence of external factors - such as exams - in the results. The third and fourth training sessions were then held, with a week's interlude, as illustrated in figure 1.

Patients were selected by the consultant rheumatologists and split into 2 groups according to whether their pathology/ inflammation was that of a knee-related rheumatic disease [group 1] or rheumatoid arthritis (RA) [group 2]. Patient selection coincided with medical appointments that day and data gathered included age, sex, medication, and disease duration.

Patients in their respective groups underwent examination by all 4 students, one by one. Students introduced themselves to the patients and started the palpation of either the knee [group 1] or MCP [group 2] joints, recording on a data collection sheet whether there was inflammation or not. It is important to note that there was no communication between the students during the process so as to avoid any potential results bias.

Immediately after the clinical examination performed by the 4 students, a third, senior consultant rheumatologist (EN) (different from the training session rheumatologists), with 20 years of experience in MSUS and blinded to students' results, performed an US examination of the knee [group 1] and MCP [group 2] joints of patients to detect the presence of B-mode synovitis, i.e. joint inflammation. This US examination was carried out in the presence of the students and the above ultrasonographer explained the anatomic structures and told the students the findings and the location of the synovitis if present.

The US examination consisted of a systematic longitudinal and transverse multiplanar examination of knee [group 1] and MCP joints [group 2] using a real-time scanner (Logiq E9, GE Medical Systems Ultrasound and Primary Care Diagnostics, Wauwatosa, WI, USA) equipped with a multi-frequency linear matrix array transducer (6-15 MHz). Machine settings were optimized before the study and standardized for the whole study. 
For the knee, the following recesses were examined: suprapatellar, parapatellar medial and lateral. For the MCP joints, the dorsal recess was assessed.

Synovitis was defined under Outcome Measures in Rheumatology (OMERACT) definitions as the presence of either effusion or synovial hypertrophy [10]. Synovitis was graduated using a semi-quantitative grade scale, as follows: grade $0-$ absence, grade $1-$ mild, grade $2-$ moderate, grade 3 - marked [11].

Students were then given the opportunity to clinically re-explore the joints so as to hone their clinical skills, whilst using feedback from ultrasound results as a reference.

\section{Statistical analysis}

Concordance between the clinical detection of arthritis by the students and the presence of ultrasound joint inflammation was analysed by Cohen's unweighted kappa. The US score of synovitis was dichotomized as follows: Grades 0 and 1, absence of arthritis; grades 2 and 3, presence of arthritis. The interobserver agreement between the students was evaluated by the Fleiss kappa test. The kappa values were considered poor if $<0-0.2$, modest if $>0.20-0.40$, moderate if $>0.40-0.60$, good if $>0.60-0.80$ and excellent if $>0.80$ [12]. Any $p$ value $<0.05$ was considered significant.

\section{Results}

\section{Patient characteristics}

Group 1.Thirty patients were included with a mean (SD, range) age of $58.5(18.0,25-89)$ years, $10(33.3 \%)$ being male, with a mean (SD, range) disease duration of $9.9(6.9,1-25)$ years and the following diagnosis: 16 with rheumatoid arthritis (RA) (53.3\%), $5(16.7 \%)$ with osteoarthritis, $2(6.7 \%)$ with connective tissue disease, $6(20.0 \%)$ with spondyloarthropathy, and 1 (3.3\%) with gout.

Eleven patients $(36.7 \%)$ received synthetic diseasemodifying anti-rheumatic drugs (DMARDs), 6 (20.0\%) biological DMARDs, 3 (10.0\%) synthetic and biologi- cal DMARDs, 2 (6.7\%) anti-hyperuricemic drugs, and 8 $(26.7 \%)$ did not receive any treatment.

Out of the 60 knees studied, 7 were explored in session I; 7 in session II; 8 in session III; and 8 in session IV.

Group 2. Eleven patients with RA were included with a mean (SD, range) age of $70.8(8.3,54-81)$ years and with a mean (SD, range) disease duration of 157 (125.2, 13-408) months.

One $(9.1 \%)$ patient did not receive any DMARDs, 4 (36.4\%) received synthetic DMARDs, 3 (27.3\%) received biological DMARDs and $3(27.3 \%)$ received synthetic and biological DMARDs. Three $(27.3 \%)$ were receiving non-steroidal anti-inflammatory drugs (NSAIDs), 2 $(18.2 \%)$ corticosteroids and another $2(18.2 \%)$ were undergoing NSAIDs plus corticosteroid therapy.

Out of the 11 patients studied, 2 were explored in session I, 3 in session II, 3 in session III and 3 in session IV.

\section{Clinical and US findings}

Group 1. The students explored 14 knees during session I, 14 knees in session II, 16 knees in session III, and 16 knees in session IV. All knees (a total of 60) were examined by US.

Group 2. The students explored $20 \mathrm{MCP}$ joints during session I, $30 \mathrm{MCP}$ joints session II, $30 \mathrm{MCP}$ joints session III, and $30 \mathrm{MCP}$ joints session IV. All MCP joints (a total of 110) were examined by US.

The prevalence of arthritis detected by the students by clinical examination is shown in table I.

\section{Agreement between students and US findings}

Group 1. Table II displays the agreement between the presence of student-detected knee arthritis by clinical examination in the four sessions and the presence of US-detected synovitis. The kappa values increased in the session III and especially in the session IV. There was a significant improvement in the agreement between session I and session III ( $\mathrm{p}=0.017)$, session I and session IV $(\mathrm{p}=0.005)$, session II and session IV $(\mathrm{p}=0.001)$, and session III and session IV $(\mathrm{p}=0.01)$.

Table I. Prevalence of student-detected arthritis.

\begin{tabular}{|c|c|c|c|c|c|c|c|c|c|}
\hline \multirow[t]{2}{*}{ Std 1} & & \multicolumn{4}{|c|}{ GROUP 1} & \multicolumn{4}{|c|}{ GROUP 2} \\
\hline & & Std 2 & Std 3 & Std 4 & Std 1 & Std 2 & Std 3 & Std 4 & \\
\hline Session & Arthritis & n (\%) & n (\%) & n (\%) & n (\%) & n (\%) & n (\%) & n (\%) & n (\%) \\
\hline \multirow[t]{2}{*}{$\mathrm{I}$} & Yes & $8(57.1)$ & $7(50)$ & $5(35.7)$ & $7(50)$ & $4(20)$ & $3(15)$ & $4(20)$ & $4(20)$ \\
\hline & No & $6(42.9)$ & $7(50)$ & $9(64.3)$ & $7(50)$ & $16(80)$ & $17(85)$ & $16(80)$ & $16(80)$ \\
\hline \multirow[t]{2}{*}{ II } & Yes & $6(42.9)$ & $6(42.9)$ & $6(42.9)$ & $7(50)$ & $15(50)$ & $15(50)$ & $22(73.3)$ & $18(60)$ \\
\hline & No & $8(57.1)$ & $8(57.1)$ & $8(57.1)$ & $7(50)$ & $15(50)$ & $15(50)$ & $8(26.7)$ & $12(40)$ \\
\hline \multirow[t]{2}{*}{ III } & Yes & $6(37.5)$ & $7(43.8)$ & $6(37.5)$ & $6(37.5)$ & $9(30)$ & $15(50)$ & $19(63.3)$ & $19(63.3)$ \\
\hline & No & $10(62.5)$ & $9(56.3)$ & $10(62.5)$ & $10(62.5)$ & $21(70)$ & $15(50)$ & $11(36.7)$ & $11(36.7)$ \\
\hline \multirow[t]{2}{*}{ IV } & Yes & $3(18.8)$ & $2(12.5)$ & $5(31.3)$ & $2(12.5)$ & $9(30)$ & $11(36.7)$ & $14(46.7)$ & $12(40)$ \\
\hline & No & $13(81.3)$ & $14(87.5)$ & $11(68.8)$ & $14(87.5)$ & $21(70)$ & $19(63.3)$ & $16(53.3)$ & $18(60)$ \\
\hline
\end{tabular}

Std - student, $\mathrm{n}$ - number of examined joints 
Table II. Agreement between the presence of arthritis detected by the students (group 1) and the presence of synovitis detected by US.

\begin{tabular}{|c|c|c|c|c|c|c|c|c|}
\hline & Session I & & Session II & & Session III & & Session IV & \\
\hline Student & KAPPA (CI 95\%) & $\mathbf{p}$ & KAPPA (CI 95\%) & $\mathbf{p}$ & KAPPA (CI 95\%) & $\mathbf{p}$ & KAPPA (CI 95\%) & $\mathbf{p}$ \\
\hline 1 & $-0.12(-0.62-0.29)$ & 0.64 & $0.44(-0.01-0.81)$ & 0.086 & $0.20(-0.29-0.60)$ & 0.424 & $0.59(0.0$ & 0.018 \\
\hline 2 & $-0.29(-0.78-0.12)$ & 0.28 & $-0.12(-0.62-0.29)$ & 0.64 & $0.36(-0.11-0.73)$ & 0.152 & $0-0.89)$ & 0.226 \\
\hline 3 & $-0.04(-0.56-0.38)$ & 0.872 & $0.16(-0.33-0.56)$ & 0.533 & $0.20(-0.29-0.60)$ & 0.424 & $0.67(0.27-1.00)$ & 0.004 \\
\hline 4 & $0.14(-0.35-0.55)$ & 0.577 & $0.29(-0.21-0.69)$ & 0.28 & $0.47(0.02-0.83)$ & 0.062 & $0.77(0.33-1.00)$ & 0.002 \\
\hline
\end{tabular}

Table III. Agreement between the presence of arthritis detected by the students (group 2) and the presence of synovitis detected by US.

\begin{tabular}{|c|c|c|c|c|c|c|c|c|}
\hline & Session I & & Session II & & Session III & & Session IV & \\
\hline Student & KAPPA (CI 95\%) & $\mathbf{p}$ & KAPPA (CI 95\%) & $\mathbf{p}$ & KAPPA (CI 95\%) & $\mathbf{p}$ & KAPPA (CI 95\%) & $\mathbf{p}$ \\
\hline 1 & $0.04(-0.33-0.42)$ & 0.822 & $-0.07(-0.42-0.28)$ & 0.713 & $0.25(-0.10-0.61)$ & 0.16 & $0.44(0.12-0.75)$ & 0.013 \\
\hline 2 & $0.14(-0.20-0.48)$ & 0.413 & $-0.20(-0.55-0.57)$ & 0.269 & $0.20(-0.14-0.54)$ & 0.256 & $0.59(0.29-0.88)$ & 0.001 \\
\hline 3 & $-0.17(-0.52-0.18)$ & 0.369 & $0.06(-0.23-0.35)$ & 0.697 & $0.25(-0.04-0.54)$ & 0.11 & $-0.01(-0.37-0.35)$ & 0.961 \\
\hline 4 & $0.26(-0.12-0.63)$ & 0.178 & $0.29(-0.04-0.61)$ & 0.098 & $0.38(0.10-0.65)$ & 0.017 & $0.66(0.39-0.93)$ & $<0.001$ \\
\hline
\end{tabular}

Table IV. Agreement between students [group 1].

\begin{tabular}{lll}
\hline SESSION & KAPPA (CI 95\%) & p \\
\hline I & $0.55(0.33-0.76)$ & $<0.0005$ \\
II & $0.54(0.33-0.76)$ & $<0.0005$ \\
III & $0.63(0.43-0.83)$ & $<0.0005$ \\
IV & $0.25(0.05-0.45)$ & 0.008 \\
\hline
\end{tabular}

Table V. Agreement between students [group 2].

\begin{tabular}{lll}
\hline SESSION & KAPPA (CI 95\%) & p \\
\hline I & $0.15(0.07-0.33)$ & 0.048 \\
II & $0.25(0.10-0.39)$ & $<0.001$ \\
III & $0.31(0.16-0.46)$ & $<0.001$ \\
IV & $0.39(0.24-0.54)$ & $<0.001$ \\
\hline
\end{tabular}

Group 2. Table III displays the agreement between the presence of student-detected MCP arthritis and the presence of US-detected synovitis over the 4 sessions. There seems to be a slight increase in the kappa values in session III. However, three out of 4 students produced clearly better results in session IV. There was a significant improvement in the agreement between session I and session III ( $\mathrm{p}=0.024)$, session I and session IV $(\mathrm{p}=<0.001)$, session II and session IV ( $\mathrm{p}=0.002)$ and session III and session IV $(\mathrm{p}=0.013)$.

\section{Agreement between students}

Group 1. The agreement between students improved from session I to session III, being good in the latter. However, less agreement was observed in the last session (Table IV).

Group 2. The agreement between students improved from session I to session IV (Table V).

\section{Discussions}

US has been used in various studies to improve medical students' clinical examination skills [13-16]. The majority of the studies focused on students' skills to detect liver margins $[13,14]$, inferior limit of the lung or thyroid size [15], or to localize femoral artery and vein [16].

The results of our study showed that medical students significantly improved their skills in the detection of synovitis in target joints using US as an educational tool for feedback. Overall, interobserver agreement improved over the duration of the training course proving no worse than that reported from expert physicians in the field [1$3]$.

The main weakness of the study is the lack of group control. This means that we cannot be sure that clinical examination improvement was only due to the US feedback; results may have been similar even without US. Additionally, US joint inflammation scores were dichotomised. The argument for considering grade 0 and 1 of US synovitis as absence of clinical inflammation is that both can be observed in normal joints [17]. Another weakness of this study is that we did not assess the student's opinion regarding potential self-confidence improvement or educational value of the training program. On the other hand, one of the study strengths was that the students were unaware of their colleagues' evaluation and the ultrasonographer was unaware of the students' evaluation. Additionally, students reached levels of interobserver reliability when assessing joints similar to that of their more experienced, veteran colleagues, experts in the musculoskeletical field [1-3], although a larger group of patients would be required to substantiate this further. 
MSUS is a user-friendly, non-invasive, objective and readily-available technique which can be used to improve students' clinical skills in the detection of synovitic joints. The technique offers immediate feedback meaning the patient can be re-examined on the spot if necessary. The training was overseen by a sonographer with over 20 years' experience and, last but not least, the cost of this training was affordable.

Although we did not formally assess the student's feedback, at the end of the study they declared to have greatly appreciated the educational value of this innovative learning method.

In conclusion, MSUS may be an effective technique for helping students to acquire the ability to detect joint inflammation. Additionally, we all found the practical training extremely useful and would encourage those who prepare the medical curricula to consider its inclusion.

\section{Conflict of interest: none}

\section{References}

1. Felson DT, Zhang Y. An update on the epidemiology of knee and hip osteoarthritis with a view to prevention. Arthritis Rheum 1998; 41: 1343-1355.

2. Carmona L, Ballina J, Gabriel R, Laffon A; EPISER Study Group. The burden of musculoskeletal diseases in the general population of Spain: results from a national survey. Ann Rheum Dis 2001; 60: 1040-1045.

3. Cheung PP, Gossec L, Mak A, March L. Reliability of joint count assessment in rheumatoid arthritis: a systematic literature review. Semin Arthritis Rheum 2014; 43 721-729.

4. Kane D, Balint PV, Sturrock RD. Ultrasonography is superior to clinical examination in the detection and localization of knee joint effusion in rheumatoid arthritis. J Rheumatol 2003; 30: 966-971.

5. Karim Z, Wakefield RJ, Quinn M, et al. Validation and reproductibility of ultrasonography in the detection of syno- vitis in the knee: a comparison with arthroscopy and clinical examination. Arthritis Rheum 2004; 50: 387-394.

6. Swamy M, Searle RF. Anatomy teaching with portable ultrasound to medical students. BMC Med Educ 2012; 12: 99.

7. Stringer MD, Duncan LJ, Samalia L. Using real-time ultrasound to teach living anatomy: an alternative model for large classes. N Z Med J 2012; 125: 37-45.

8. Jurjus RA, Dimorier K, Brown K, et al. Can anatomists teach living anatomy using ultrasound as a teaching tool? Anat Sci Educ 2014; 7: 340-349.

9. Cheung PP, Lahiri M, Teng GG, et al. A randomized controlled trial for improving patient self-assessment of synovitis in rheumatoid arthritis with education by ultrasonography: the RAEUS Study. Rheumatology 2015; 54: 1161-1169.

10. Wakefield RJ, Balint PV, Szkudlarek M, et al; OMERACT 7 Special Interest Group. Musculoskeletal ultrasound including definitions for ultrasonographic pathology. J Rheumatol 2005; 32: 2485-2487.

11. Naredo E, Rodríguez M, Campos C, et al. Validity, reproducibility and responsiveness of a 12 -joint simplified power Doppler ultrasonographic assessment of joint inflammation in rheumatoid arthritis. Arthritis Rheum 2008; 59: 512-522.

12. Landis JR, Koch GG. The measurement of observer agreement for categorical data. Biometrics 1977; 33: 159-174.

13. Barloon TJ, Brown BP, Abu-Yousef MM, et al. Teaching physical examinationof the adult liver with use of real-time sonography. Acad Radiol 1998; 5: 101-103.

14. Butter J, Grant TH, Egan M, et al. Does ultrasound training boost year medical student competence and confidence when learning abdominal examination? Med Educ 2007; 41: 843-848.

15. Fodor D, Badea R, Poanta L, Dumitrascu DL, Buzoianu $\mathrm{AD}$, Mircea PA. The use of ultrasonography in learning clinical examination: a pilot study involving third year medical students. Med Ultrason 2012; 14: 177-181.

16. Ahn JS, French AJ, Thiessen ME, et al. Using ultrasound to enhance medical students' femoral vascular physical examination skills. J Ultrasound Med 2015; 34: 1771-1776.

17. Witt M, Mueller F, Nigg A, et al. Relevance of grade 1 grayscale ultrasound findings in wrists and small joints to the assessment of subclinical synovitis in rheumatoid arthritis. Arthritis Rheum 2013; 65: 1694-1701. 